\title{
Estimulación Eléctrica en Niños y Adolescentes Con Parálisis Cerebral: Una Revisión Sistemática
}

\author{
Electrical Stimulation In Children And Adolescents With Cerebral Palsy: \\ A Systematic Review
}

\author{
Raul Sulla-Torres, Ruben Vidal-Espinoza, Jaime Pacheco-Carrillo, Jorge Apaza-Cruz, Jose Sulla-Torres, \\ Cristian Luarte-Rocha, ${ }^{6}$ Marco Cossio-Bolaños, Rossana Gomez-Campos ${ }^{7}$
}

\section{Resumen}

Objetivos: Analizar los rangos de edad más investigados en niños con parálisis cerebral (PC). Identificar las regiones anatómicas tratadas mediante electro-estimulación (EE). Determinar la frecuencia y duración de los protocolos utilizados en los estudios efectuados entre el 2009 al 2019.

Metodología: Se efectuó un estudio bibliográfico de revisión sistemática sobre el uso de EE en niños con PC en los últimos 10 años. Se consideró la búsqueda en la base de datos Pub Med. Se utilizó la técnica de la observación para extraer la información y se registraron los indicadores en una ficha de evaluación (tipo de estudio, muestra, región anatómica tratada, sujetos investigados, frecuencia y duración de las terapias).

Resultados: La mayoría de los estudios han desarrollado los tratamientos con EE en las piernas y en los extensores del codo y la muñeca y un estudio -al mismo tiempo- en rostro y piernas. Las edades investigadas oscilan desde 1 hasta los 16 años. Los protocolos han sido utilizados desde 3 hasta 5 veces por semana, y van desde 2 semanas hasta 12 semanas. El tiempo de administración de la EE osciló entre 20 a 60 minutos.

Conclusión: Para conseguir beneficios positivos es necesario desarrollar programas desde 3-5 veces por semana y cada sesión en periodos de 20 a 60 minutos. Esta sistematización podría contribuir a una organización eficaz en futuros programas de intervención y en la estimulación de otras regiones anatómicas poca exploradas.

Palabras clave: Estimulación eléctrica, parálisis cerebral, intervenciones, niños y adolescentes.

\section{Abstract}

Objectives: To analyze the most investigated age ranges in children with cerebral palsy. To identify the anatomical regions treated by electro stimulation. To determine the frequency and duration of the protocols used in the studies carried out between 2009 to 2019 .

Methodology: A systematic review of the literature on the utilization of electro stimulation in children with cerebral palsy in the last 10 years was carried out. The search was considered in the Pub Med database. The observation technique was used to extract the information and the indicators were recorded in an evaluation form (type of study, sample, anatomical region treated, subjects investigated, frequency and duration of therapies).

Results: Most of the studies have developed the treatments with electro stimulation in the legs and extensors of the elbow and the wrist and one study -at the same time- in the face and legs. The ages investigated ranged between 1 and 16 years. The protocols have been used from 3 to 5 times per week and in a range of 2 to 12 weeks. The time of administration of the electro stimulation ranged between 20 to 60 minutes.

Conclusion: To obtain positive benefits it is necessary to develop programs from 3-5 times per week and each session in periods of 20 to 60 minutes. This systematization could contribute to an efficient organization in future intervention programs and in the stimulation of other anatomical regions less explored.

Keywords: Electrical stimulation, cerebral palsy, interventions, children and adolescents.

Rev. Ecuat. Neurol. Vol. 29, $\mathrm{N}^{\circ} 1,2020$

\footnotetext{
'Universidad Católica Santa María, Arequipa, Perú

${ }^{2}$ Universidad Católica Silva Henriquez, Santiago, Chile

${ }^{3}$ Universidad del Bio Bio, Chillán, Chile

${ }^{4}$ Departamento Académico de Ingeniería Electrónica, facultad de Ingeniería Mecánica Eléctrica, Electrónica y Sistemas, Universidad Nacional del Altiplano, Puno, Perú

${ }^{5}$ Universidad Nacional de San Agustín, Arequipa, Perú.

${ }^{6}$ Universidad San Sebastián, Concepción, Chile

Universidad Católica del Maule, Talca, Chile
}

Correspondencia:

Rossana Gómez-Campos

Avenida San Miguel № 3605

Facultad de Ciencias de la Educación

Universidad Católica del Maule. Talca, Chile

E-mail: rossaunicamp@gmail.com 


\section{Introducción}

La parálisis cerebral (PC) se caracteriza por la debilidad muscular, alteración de la coordinación de los músculos y espasticidad de las extremidades. Además, son acompañados por alteraciones de la sensibilidad, cognición, comunicación, percepción, trastornos de la conducta y convulsiones?

Este trastorno neurológico pediátrico está fuertemente asociado con cuestiones de salud pública e inclusive con la presencia de múltiples incapacidades y diversos niveles de dependencia. Estos pacientes muestran una gran heterogeneidad (por ejemplo, diplejía, hemiplejia, marcha atáxica y marcha espástica, todos con síntomas más o menos graves). ${ }^{4}$

De hecho, la investigación de la PC en los últimos años se ha profundizado en niños y adolescentes, especialmente en programas de intervención $)^{5-8}$ puesto que la meta de cualquier programa de tratamiento para la PC es maximizar la función y minimizar el desarrollo de problemas secundarios, disminuir el número de caídas, mejorar la participación en actividades con otras personas, mejorar su apariencia estética, desarrollar sus habilidades cognitivas, habilidades sociales, estado emocional e independencia funcional?

En ese sentido, la EE es considerada una técnica mínimamente invasiva, la que puede ser utilizada desde una perspectiva funcional, neuromuscular y terapéutica. ${ }^{10}$ Esta consiste en aplicar una corriente eléctrica de intensidad suficiente para provocar estimulación funcional, neuromuscular y estimulación terapéutica." Por lo general se utilizan para modificar los impedimentos y las limitaciones de la actividad muscular en niños con PC.

En consecuencia, ante la necesidad de tratar la alta frecuencia y los múltiples problemas que caracterizan a los niños con PC, como por ejemplo, mejorar la rigidez y reflejos exagerados (espasticidad), estimular los músculos antagónicos, fortalecer los músculos en general y mejorar el tiempo de movimiento coordinado, ${ }^{11}$ es necesario que los profesionales de las ciencias de la salud y los investigadores en general asuman estos desafíos con el propósito de mejorar el estado de salud de esta población altamente vulnerable.

En consecuencia, conocer los grupos etarios más estudiados, las regiones anatómicas estimuladas y la frecuencia y duración de los tratamientos desarrollados por medio de EE en niños con PC podrían contribuir en la planificación de acciones que tengan que ver con la implementación de futuros programas de intervención.

Por lo tanto, los objetivos del estudio son: a) analizar los rangos de edad más investigados, b) identificar las regiones anatómicas tratadas mediante electro-estimulación y c) determinar la frecuencia y duración de los protocolos utilizados en los estudios efectuados entre el 2009 al 2019 en la base de datos Pub Med.

\section{Métodos}

\section{Tipo de estudio}

Se efectuó un estudio bibliográfico de revisión sistemática sobre el uso de EE en niños con PC en los últimos 10 años. Se consideró la búsqueda de información desde los años 2009-2019. Todos los estudios están publicados en idioma inglés que se han efectuado en todo el mundo. Las palabras clave utilizadas fueron: electric stimulation, vest, cerebral palsy, children. Se excluyeron investigaciones que fueron desarrolladas fuera del rango de años considerado, incluyendo hasta el 30 de abril 2019. Se excluyeron los estudios que no habían utilizado ningún tipo de EE.

\section{Técnicas e instrumentos}

Se utilizó la técnica de la observación para sistematizar los artículos originales. Se elaboró una ficha de observación para registrar la información. Los indicadores utilizados fueron, tipo de estudio, muestra, región anatómica tratada, sujetos investigados, frecuencia y duración de las terapias.

\section{Procedimiento de búsqueda bibliográfica}

Se utilizó la base de datos de Biblioteca Nacional de Medicina de los Estados Unidos denominada Pub Med (https://www.ncbi.nlm.nih.gov/pubmed/), cuyas temáticas están asociadas al campo de las ciencias de la salud. Este periodo de búsqueda de información tuvo como duración una semana desde el 1 al 19 de mayo 2019. La mejor información de búsqueda de coincidencia fue: MeSH Terms: cerebral palsy; research design; electric stimulation; child.

El proceso de selección de estudios se basó en las sugerencias descritas por Liberati et $\mathrm{al}^{12}$ siguiendo las cuatro fases del flujograma denominado PRISMA. La figura 1 muestra todo el proceso desarrollado.

En la primera etapa se identificó un total de 26 artículos a nivel internacional (Pub Med), los que fueron considerados como posibles estudios potenciales para la sistematización. En la segunda etapa (tamizaje) se procedió a la lectura del total de los resúmenes y la metodología de los estudios. En la tercera etapa se identificaron los estudios como elegibles los que pasaron a ser incluidos $(\mathrm{n}=15)$. En general, se incluyeron los estudios que tenían que ver con las palabras claves utilizadas en el estudio y un rango de edad máximo de 16 años, los que al final se redujeron a nueve estudios. Se excluyeron los estudios mixtos (cualitativos y cuantitativos) y revisiones sistemáticas y de meta-análisis. 


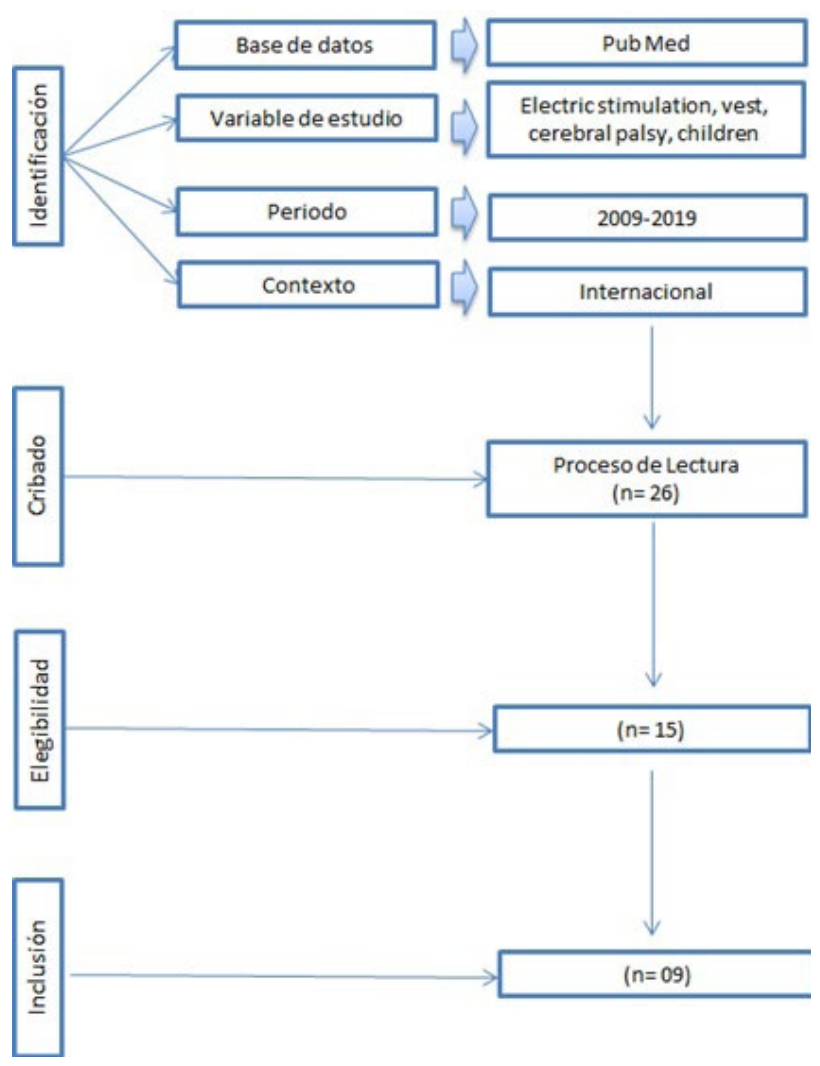

Figura 1. Diagrama de flujo PRISMA para sistematización de artículos originales 2009-2019.

\section{Análisis de estudios}

El proceso de organización de los datos de cada uno de los artículos se digitó en plantillas de Excel. A partir de ello, se sistematizó la información por medio de análisis cualitativos y/o cuantitativos. Se describieron los datos en tablas y se organizaron por medio de frecuencias (f). Estos indicadores permitieron cuantificar de forma descriptiva los nueve estudios según año, tamaño de muestra, región anatómica tratada, rango de edad, y protocolo utilizado (frecuencia y duración).

\section{Resultados}

Los estudios efectuados desde 2009 hasta 2019 se encuentran descritos en la tabla 1. Los nueve estudios han desarrollado estudios experimentales (programas de intervención) utilizado EE para tratar a niños y adolescentes con PC en una región anatómica especifica.

En general, la mayoría de los estudios han desarrollado los tratamientos con EE en las piernas (gastrocnemio $(\mathrm{n}=4)$ y en los extensores del codo y la muñeca $(n=4)$ y un estudio en el rostro y al mismo tiempo en las piernas.

Los rangos de edad investigados durante los años 2009 a 2019 se muestran en la figura 2. Las edades investigadas según los nueve estudios oscilan desde 1 hasta los 16 años. Nótese que 5 estudios han efectuado sus investigaciones en niños comprendidos desde 1 hasta los 6 años. Los otros 4 estudios han investigado en edades superiores hasta los 11, 14 y 16 años, respectivamente.

En cuanto a los protocolos utilizados para las terapias con EE se muestran en la figura 3, que indica que han sido utilizados desde 3 hasta 5 veces por semana, y van desde 2 semanas hasta 12 semanas en su mayoría, salvo el estudio Xu et al6 que efectuó 48 semanas. En general, el tiempo de administración de la $\mathrm{EE}$ en los niños con PC osciló entre 20 a 60 minutos.

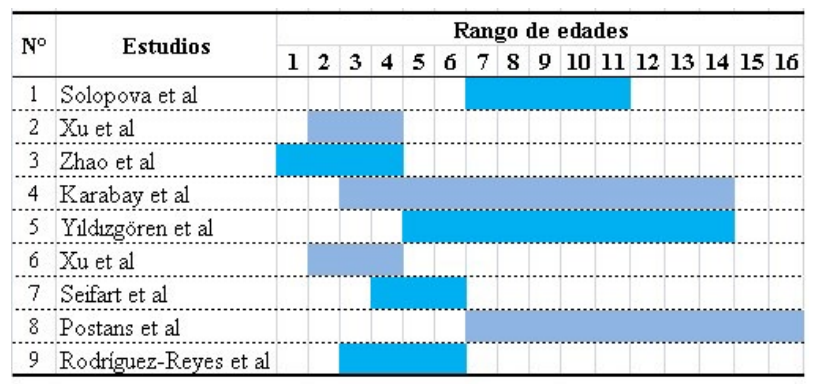

Figura 2. Rango de edades utilizadas por las investigaciones 20092019 que aplican terapias de EE con PC

Tabla 1. Estudios experimentales que han utilizado electro-estimación en niños y adolescentes con parálisis cerebral.

\begin{tabular}{|llllll|}
\hline $\mathbf{N}^{\circ}$ & Autores & Año & Tipo de estudio & Tamaño Muestra & Región Anatómica tratada \\
\hline 01 & Solopova et al & 2017 & Experimental & $\mathrm{GE}=13 / \mathrm{GC}=15$ & Femur y Gastrocnemio \\
\hline 02 & Xu et al & 2015 & Experimental & $\mathrm{GE}=23 / \mathrm{GE}=22 / \mathrm{GE}=22$ & Brazo y Manos \\
\hline 03 & Zhao et al & 2015 & Experimental & $\mathrm{G} . \mathrm{Es}=21 / \mathrm{G}$. SE $=19$ & Rostro y Piernas (gastrocnemio) \\
\hline 04 & Karabay et al & 2015 & Experimental & $\mathrm{GE}=14 / \mathrm{GC}=14$ & Gastrocnemio \\
\hline 05 & Ylldızgören et al & 2014 & Experimental & $\mathrm{GE}=12 / \mathrm{GC}=12$ & Muñeca \\
\hline 06 & Xu et al & 2012 & Experimental & $\mathrm{GE}=13 / \mathrm{GC}=15$ & Muñeca \\
\hline 07 & Seifart et al & 2010 & Experimental & $\mathrm{GE}=08 / \mathrm{GC}=07$ & Piernas Gastrocnemio \\
\hline 08 & Postans et al & 2010 & Experimental & $\mathrm{GE}=6$ & Muñeca y codo \\
\hline 09 & Rodriguez-Reyes et al & 2009 & Experimental & $\mathrm{GE}=10$ & Muñeca y Codo \\
\hline
\end{tabular}

Leyenda: GE: Grupo experimental, GC: Grupo Control, Es: Espasmo, SE: Sin espasmo. 
Figura 3. Protocolos utilizados para EE en niños y adolescentes con PC durante los años 2009-2019.

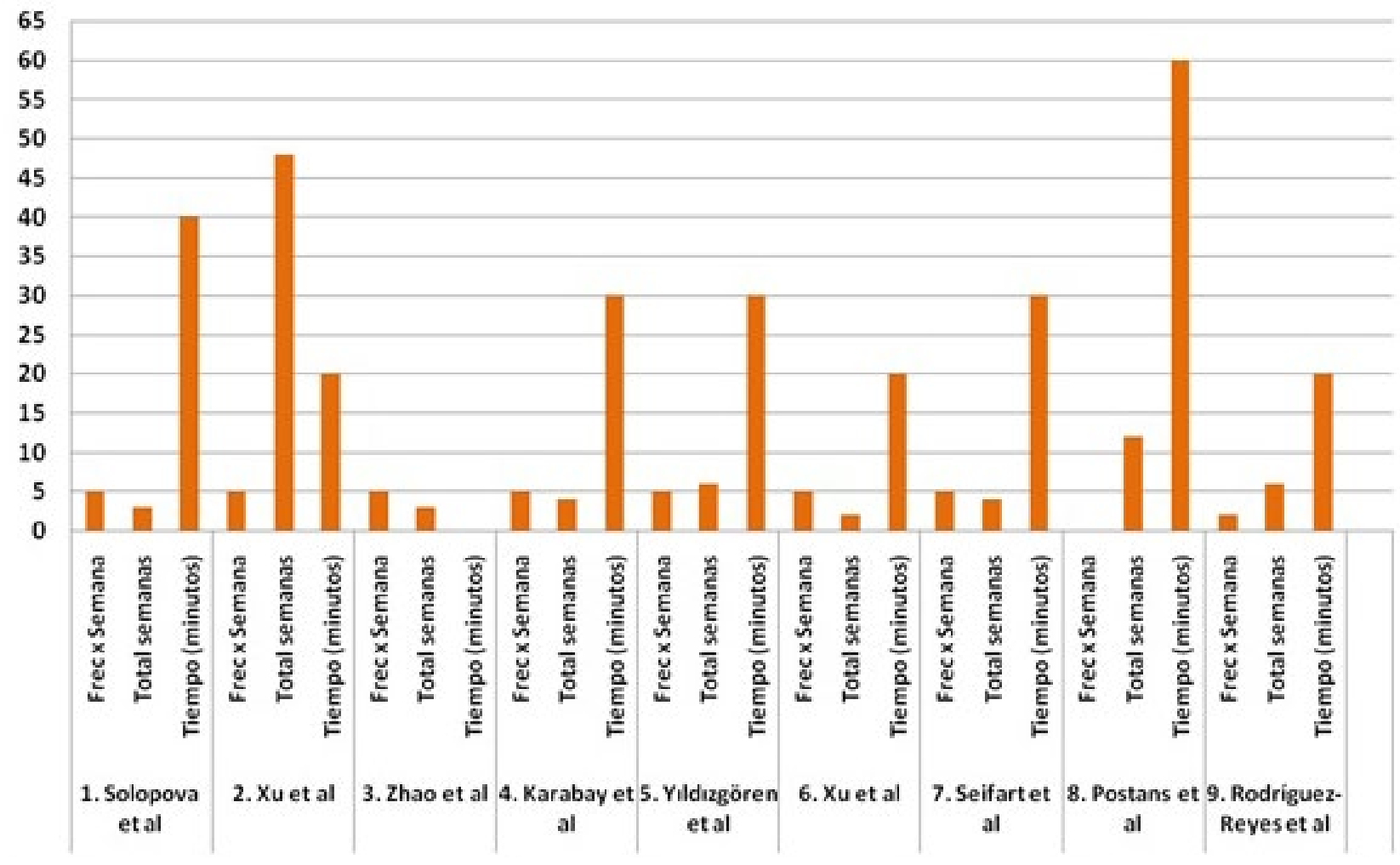

\section{Discusión}

Los resultados del estudio han demostrado que en los 10 últimos años (Pub Med) el rango de edad más investigado oscila dese 1 hasta los 16 años. Además 5 estudios de los 9 identificados han considerado en sus muestras a niños de ambos sexos de 1 hasta los 6 años, inclusive hay dos estudios que han incluido edades tempranas y avanzadas.

Estas preferencias podrían deberse al hecho que los programas de intervención en muestras con PC por lo general se deben realizar precozmente, puesto que esto podría ayudar a estimular más rápidamente el cerebro inmaduro13 y minimizar deficiencias motoras e incapacidades comúnmente encontradas en esta población. ${ }^{14}$

De hecho, los estudios sistematizados en general se han centrado en su mayoría a mejorar no sólo las funciones corporales y habilidades funcionales, sino en optimizar la coordinación, contracturas, el dolor, el estrés y la sensibilidad. $^{5-8,15-19}$

En general, investigar en niños y adolescentes en fase de crecimiento es relevante, y mejor aun cuando se trata de edades tempranas, ya que los niños tienen la posibilidad de recibir estimulación y atención adecuada y a tiempo, con lo cual, es posible mejorar la independencia funcional, aliviar la carga familiar y consecuentemente la calidad de vida.

Este tipo de intervenciones favorece a los niños para que puedan integrarse y desarrollarse dentro del medio familiar y social con más facilidad, ${ }^{20} \sin$ embargo, es necesario destacar que cuando se trabaja con niños con PC se debe considerar a la familia, puesto que juega un papel relevante en el inicio de las terapias, organización de los planes, esperanza y visión de futuro.1

En cuanto a las regiones anatómicas de EE, los resultados sistematizados mostraron que los nueve artículos reportaron beneficios positivos en niños con PC, en especial cuando han sido estimulados específicamente en el gastrocnemio, músculos faciales, los extensores del codo y la muñeca- ${ }^{5-815-19}$ Estas evidencias reflejan en general que las regiones anatómicas preferidas por la mayoría de los estudios fueron las extremidades superiores e inferiores.

Estos resultados observados hacen suponer que los programas de intervención desarrollados están interesados en el control y reflejo de enderezamiento, mejoramiento de la marcha cuando se estimulan las extremidades inferiores ${ }^{10,22}$ y por el contario, cuando se estimulan las extremidades superiores, los programas están orientados al fortalecimiento muscular e incremento de rango articular de forma general ${ }^{23}$ y más específicamente para mejorar las funciones como sujetar, agarrar, mover los brazos y soltar objetos. ${ }^{24}$

$\mathrm{La} \mathrm{EE}$ en niños con PC, tanto en las extremidades superiores, como en inferiores podría jugar un papel relevante en el presupuesto familiar, ${ }^{25}$ siempre que exista la iniciativa de instituciones que promocionen estos programas de intervención para las familias con miembros que padecen de PC. 
Esta política podría contribuir al desarrollo de la autonomía, al mejoramiento de la convivencia familiar y social, y a la adopción de una rutina diaria que facilite la interacción con el entorno donde se desarrollan. ${ }^{26}$

Respecto a la frecuencia y duración de las intervenciones desarrolladas, las nueve investigaciones han evidenciado resultados alentadores en sus diseños experimentales (aleatorizados), puesto que, a pesar de presentar diversas metodologías para la localización de los puntos anatómicos, intensidad, y duración en los tiempos de tratamiento (extremidades superiores e inferiores), han reportado resultados positivos en niños y adolescentes con PC.

Estos programas de intervención por medio de EE en su mayoría se realizó entre 20 a 60 minutos por sesión y por lo general fueron efectuadas desde 3 a 5 veces por semana. Las duraciones de los programas fueron desde las dos hasta las 12 semanas en su mayoría, aunque hubo un estudio que consideró hasta 48 semanas.

En general, el tiempo de la EE de forma integral según Díaz-Reina ${ }^{27}$ es alrededor de los 20-30 minutos, aunque De la Cámara, $\operatorname{Pardos}^{28}$ considera 20 minutos aproximadamente. Tiempos de más minutos podrían ocasionar problemas metabólicos en la musculatura y ocasionaría fatiga e incomodidad en los niños.

Además, la frecuencia semanal varió de tres a seis veces por semana, hasta un máximo de seis a ocho semanas.$^{29}$ De hecho, los resultados de las investigaciones son muy variados, lo que definitivamente impide estandarizar la frecuencia y duración. Lo que si parece ser evidente es que las intervenciones y/o terapias en los niños con PC no deben ser inferiores a tres veces por semana, cuyos estímulos podrían oscilar entre 20-30 minutos por día. La extensión del programa va depender de las condiciones en que se desarrollen y podrían extenderse hasta 12 semanas aproximadamente.

En suma, esta investigación evidenció información relevante para que futuros estudios puedan diseñar programas de intervención en niños y adolescentes con PC, independientemente del tipo de EE. ${ }^{4}$ Por ejemplo, la estimulación funcional (para estimular la superficie de los músculos y/o nervios que tienen un control motor), la estimulación neuromuscular (estimula la superficie de los músculos que generalmente es de alta intensidad y la duración corta para el propósito de iniciar una contracción y un movimiento subsiguiente) y la estimulación terapéutica (niveles por debajo del umbral) que se puede aplicar de forma continua durante un largo período de tiempo). Es ampliamente utilizada y difundida por los profesionales de la fisioterapia y la rehabilitación en diversos tratamientos, que van desde la reparación de tejidos, entrenamiento en pacientes, aumento de fuerza, alivio del dolor (analgésico), mejoramiento de la apariencia estética, desarrollar sus habilidades cognitivas, habilidades sociales, estado emocional e independencia funcional., ${ }^{90-32}$
Algunas limitaciones deben ser descritas en esta revisión. Por ejemplo, se buscó información de una sola base de datos (Pub med), esto podría limitar la cantidad de investigaciones originales efectuadas durante los últimos diez años; además, no se pudieron homogeneizar las investigaciones sistematizadas debido a la heterogeneidad de la muestra y las terapias desarrolladas según necesidades de los pacientes. Futuras revisiones deben considerar estos aspectos para especificar la información de los investigadores y profesionales de las ciencias de la salud.

En conclusión, estos resultados sugieren que para conseguir beneficios positivos en niños con PC por medio de la EE en el gastrocnemio y los extensores del codo y la muñeca es necesario desarrollar programas desde 3 hasta 5 veces por semana y cada sesión en periodos de 20 a 60 minutos. No obstante es necesario analizar a profundidad el tipo de trastorno de los pacientes, esta sistematización podría contribuir a una organización eficaz en futuros programas de intervención y en la estimulación de otras regiones anatómicas poca exploradas por los investigadores.

\section{Referencias}

1. Stackhouse SK, Binder-Macleod SA, Lee SC. Voluntary muscle activation, contractile properties, and fatigability in children with and without cerebral palsy. Muscle and Nerve 2005; 31(5): 594- 601.

2. Rosenbaum P, Paneth N, Leviton A, Goldstein M, Bax M, Damiano D, et al. A report: the definition and classification of cerebral palsy. Dev Med Child Neurol Suppl 2007; 109: 8-14.

3. Baltor MRR, Dupas G. Experiences from families of children with cerebral paralysis in context of social vulnerability. Revista Latino-Americana de Enfermagem 2013;21(4):956-63

4. Cauraugh JH, Naik SK. Children with cerebral palsy: a systematic review and meta-analysis on gait and electrical stimulation. Clinical Rehabilitation 2010; 24 : 963-978

5. Solopova IA, Sukhotina IA, Zhvansky D, Ikoeva GA, Vissarionov SV, Baindurashvili AG, Edgerton VR, Gerasimenko YP, Moshonkina TR. Effects of spinal cord stimulation on motor functions in children with cerebral palsy. Neurosci Lett. 2017; 3(639):192-198. doi: 10.1016/j.neulet.2017.01.003.

6. Xu K, He L, Mai J,Yan X, Chen Y. Muscle Recruitment and Coordination following Constraint-Induced Movement Therapy with Electrical Stimulation on Children with Hemiplegic Cerebral alsy: A Randomized Controlled Trial. PlosOne 2015; 10(10):e0138608.doi:10.1371/ journal.pone.0138608.

7. Yıldızgören MT, Nakipoğlu Yüzer GF, Ekiz T, Özgirgin $\mathrm{N}$. Effects of neuromuscular electrical stimulation on the wrist and finger flexor spasticity and hand functions in cerebral palsy. Pediatr Neurol. 2014;51(3):360-4. doi: 10.1016/j.pediatrneurol.2014.05.009. Epub 2014 May 21. 
8. Seifart A, Unger M, Burger M. Functional Electrical Stimulation to Lower Limb Muscles After Botox in Children With Cerebral Palsy. Pediatr Phys Ther 2010;22:199-206

9. Díaz-Martínez L, Arellano Saldaña M, del Valle Cabrera MG, Miranda Duarte A, Rodríguez Reyes G, Montero Aldo A. Utilidad de la estimulación eléctrica neuromuscular posterior a la aplicación de toxina botulínica en niños con hemiparesia espástica. Revista Mexicana de Medicina Física y Rehabilitación 2005; 17: 16-22

10. Postans NJ, Granat MH. Effect of functional electrical stimulation, applied during walking, on gait in spastic cerebral palsy. Dev Med Child Neurol 2005; 47: 46-52

11. Merrill DR. Review of electrical stimulation in cerebral palsy and recommendations for future directions. Developmental Medicine \& Child Neurology 2009, 51 (Suppl. 4): 154-165

12. Liberati A, Altman DG, Tetzlaff J, Mulrow C, Gøtzsche P, et al. The PRISMA statement for reporting systematic reviews and meta-analyses of studies that evaluate health care interventions: Explanation and elaboration. PLoS Med 2019;6:e1000100. doi:10.1371/ journal.pmed.1000100.

13. Lima CLA, Fonseca LF. Paralisia Cerebral: neurologia, ortopedia, reabilitação. Rio de Janeiro (RJ): Editora Guanabara Koogan; 2008.

14. Lacerda TTB, Magalhães LC. Análise da validade dos itens do Movement Assessment of Infants - MAI - para crianças pré-termo. Rev. Bras. Saude Mater. Infant 2006; 6(3):297-308.

15. Zhao X, Chen M, Du S, Li H, Li X: Evaluation of stress and pain in young children with cerebral palsy during early developmental intervention programs: a descriptive study. Am J Phys Med Rehabil 2015;94:169Y179

16. Ilkay Karabay, Tuna Go khan, Unsal-Malas F, Kara M, Tiftik T, Erso M, Ozcakar L. Short-term effects of neuromuscular electrical stimulation on muscle architecture of the tibialis anterior and gastrocnemius in children with cerebral palsy: preliminary results of a prospective controlled study. Am J Phys Med Rehabil 2015;94:728Y733

17. Xu K, Wang L, Mai J, He L. Efficacy of constraintinduced movement therapy and electrical stimulation on hand function of children with hemiplegic cerebral palsy: a controlled clinical trial. Disability \& Rehabilitation, 2012; 34(4): 337-346.

18. Postans N, Wright P, Bromwich W, Wilkinson I, Farmer S, Swain I. The combined effect of Dynamic splinting and Neuromuscular electrical stimulation in reducing wrist and elbow contractures in six children with Cerebral palsy. Prosthetics and Orthotics International, 2010; 34(1): 10-19

19. Rodríguez-Reyes G, Alessi-Montero A, Díaz-Martínez L, Miranda-Duarte A, Pérez-Sanpablo A. Botulinum Toxin, Physical and Occupational Therapy, and Neuromuscular Electrical Stimulation to Treat Spastic
Upper Limb of Children With Cerebral Palsy:A Pilot Study. Artif Organs 2010;34 (3): 230-234.

20. Gutiez P, Ruiz E. Orígenes y Evolución de la Atención Temprana. Una Perspectiva Histórica de la Génesis de la Atención Temprana en Nuestro País. Agentes Contextos y Procesos. Psicología Educativa 2012; 18(2): 107-122.

21. Rosenbaum P, King S, Law M, King G, Evans J. Family-Centred Service. Physical \& Occupational Therapy In Pediatrics. 1998;18(1):1-20

22. Semenova KA. The problem of rehabilitation treatment of children with cerebral palsy, Zh. Nevrol. Psikhiatr. im. S.S. Korsakova, 2012; 2(7): 9.

23. Alon G, Lewitt AF, McCarty PA. Functional electrical sitmulation (FES) may modify the poor prognosis of stroke survivors with severe motor loss of the upper extremity: A preliminary study. Am J Phys Med Rehabil 2008;87:627-36.

24. Powell J, Pandyan AD, Granat M, Cameron M, Stott DJ. Electrical stimulation of wrist extensors in poststroke hemiplegia. Stroke 1999;30:1384-9

25. Poletto M, Koller SH. Contextos ecológicos: promotores de resiliência, fatores de risco e de proteção. Estud Psicol. 2008;25(3):405-16. DOI: http://dx.doi. org/10.1590/S0103-166X2008000300009

26. Morilla C, Tanuri CA, Scarpellini AC, dos Santos P. Family resources and promotion of development of children with cerebral palsy. J Hum Growth Dev. 2017; 27(2): 166-174

27. Díaz-Reina D. El uso de la electroterapia en niños con parálisis cerebral, equipos utilizados y programación. Mov.Cient. 2009;3(1):82-87.

28. De la Camara, M, Pardos I. Revisión de los beneficios físicos de la electroestimulación integral. Apunts. Educación Física y Deportes 2016; 123: 28-33

29. Salazar AP, Pagnussat A, Alves Pereira G, Scopel G, Lukrafka JL. Neuromuscular electrical stimulation to improve gross motor function in children with cerebral palsy: a meta-analysis. Braz J Phys Ther. 2018, https:// doi.org/10.1016/j.bjpt.2019.01.006

30. Sluka KA, Bailey K, Bogush J, Olson R, Ricketts A. Treatment with either high or low frequency TENS reduces the secondary hyperalgesia observed after injection of kaolin and carrageenan into the knee joint. Pain. 1998; 77(1):97-102.

31. Russo TL, França CN, Castro CES, Salvini TF. Alterations of the chronaxie, reobase and accomodation in denervated skeletal muscle submitted to electrical stimulation. Rev Bras Fisioter. 2004;8(2):169-75.

32. Sheffler LR, Chae J. Neuromuscular electrical stimulation in neurorehabilitation. Muscle Nerve. 2007;35(5):562-90.

Financiamiento: A la Universidad Católica de Santa María de Arequipa por los fondos para la investigación de proyectos a través del Vicerrectorado de Investigación 